\title{
Model based estimation of the Eddy currents for the ITER tokamak
}

\author{
Alfredo Pironti ${ }^{1}$ \\ Consorzio CREATE - University of Naples Federico II \\ Via Claudio 21, 80125 Napoli, Italy \\ E-mail: pironti@unina.it
}

Roberto Ambrosino

Consorzio CREATE - University of Naples Parthenope

Centro Direzionale Isola C4, 80143 Napoli, Italy

E-mail: r.ambrosino@uniparthenope. it

\section{Massimiliano Mattei}

Consorzio CREATE - Second University of Naples

Aversa, 81031 Italy (e-mail:)

E-mail: massimiliano.matteieunina2. it

\section{Shakeib Arshad}

Fusion For Energy

c/ Josep Pla, $n^{\circ}$ 2, Torres Diagonal Litoral, Edificio B3,08019 Barcelona, Spain

E-mail: Shakeib. Arshadef4e. europa.eu

\section{Luca Zabeo}

ITER Organization

Route de Vinon sur Verdon, 13115 St Paul Lez Durance, France

E-mail: Luca.Zabeo@iter.org

Eddy currents flowing in the conductive structures surrounding the plasma in a tokamak affect magnetic measurements (magnetic fields and fluxes at given points), which are used to estimate plasma quantities such as plasma toroidal current, position and shape. Due to their spatial distribution and their fast variations, eddy currents are difficult sources to characterize. This is particularly true when magnetic sensors are outside the conductive plasma chamber implying that eddy currents flow between the so-called measurement contour and the plasma region. As a matter of fact many plasma shape identification algorithms assume the plasma current distribution as the only magnetic source located inside the measurement contour. In this paper we deal with the problem of estimating eddy currents in the ITER tokamak by means of a dynamic observer to wash out their contribution to magnetic measurements.

First EPs Conference on Plasma Diagnostics - $1^{\text {st }}$ ECPD

14-17 April 2015,

Villa Mondragone, Frascati (Rome) Italy

\footnotetext{
*The work reported was funded partially by Fusion for Energy under Grant F4E-GRT-047and by the Italian MIUR under PRIN grant 2010SPS9B3. This publication reflects the views of the authors only. Fusion for Energy cannot be held responsible for any use which may be made of the information contained herein. The views and opinions expressed herein do not necessarily reflect those of the ITER Organization.

${ }^{1}$ Speaker
} 


\section{Introduction}

The estimation of plasma parameters during a tokamak discharge is an important problem from an engineering point of view. Indeed many of these parameters need to be controlled and/or monitored in real time. In particular, from a magnetic control point of view [1], it is required to estimate within a prescribed accuracy the plasma current, position and shape. These parameters are not directly measurable, and reconstruction is mandatory to provide their control to desired values by means of a feedback system. Plasma identification is the set of actions aimed at reconstructing the main plasma parameters using measurements provided by available diagnostic systems and a priori information on plasma physics.

Typically, the plasma identification system, including the reconstruction algorithms, and the measurement systems, is designed with reference to an idealized nominal configuration; any discrepancy is then treated as a disturbance. As a consequence, the identification reliability strongly depends on the robustness of the mathematical modeling, on the quality of the measurements and on the uncertainties on physical and geometrical parameters. Modeling error sources can be plasma equation approximations, the presence of non modelled nonaxisymmetric structures, and neglecting additional magnetic sources, such as eddy currents flowing in the conducting structures surrounding the plasma.

The present paper explores the possibility to reconstruct eddy currents in the ITER tokamak, by means of a dynamic observer, and in the meanwhile to compensate for biases and drifts on the used magnetic measurements. In order to quantify the impact of the eddy currents in the identification problem, the inverse equilibrium code EFIT++ ([5][7]) has been used.

\section{The eddy current estimation procedure}

From a magnetic control point of view, a tokamak can be modeled by considering the magnetic interactions between the plasma, the control circuits and the passive conductors.

The equations describing this interaction are governed by the plasma momentum balance equation and by the Maxwell's equations in their quasi-stationary form. Under suitable assumptions, and by means of a numerical approach based on the Finite Element Method (FEM), the resulting system of free-boundary partial differential equations can be approximated by a finite set of ordinary differential equations (see [3]), that can be written

$$
\begin{aligned}
& L_{a a} \dot{I}_{a}+L_{a e} \dot{I}_{e}+L_{a w} \dot{w}+R_{a} I_{a}=V_{a} \\
& L_{e a} \dot{I}_{a}+L_{e e} \dot{I}_{e}+L_{e w} \dot{w}+R_{e} I_{e}=0 \\
& y=C_{a} I_{a}+C_{e} I_{e}+F w
\end{aligned}
$$

where $V_{a}$ is the vector of the voltages applied to the circuits (zero for passive coils); $I_{a}$ and $I_{e}$ are the vectors of the active (the currents flowing in the controlled coils) and of the passive currents (the eddy currents we want to estimate); $w$ is the vector of the plasma current distribution parameters (e.g. the currents in a finite number of filaments representing the plasma in the vacuum chamber, or the plasma magnetic moments, or finally the poloidal beta and the internal inductance); $y$ is a vector of magnetic output variables (the magnetic measurements in this paper); $R_{a}$ and $R_{e}$ are resistance matrices; $L_{a a}, L_{e e}, L_{a e}, L_{e a}, L_{a w}$, and $L_{e w}$ are self and mutual inductance matrices; finally $C_{a}, C_{e}$ and $F$ are output matrices linking the current sources to the magnetic measurements. Since the passive conducting structures are discretized with a 
FEM approach, the number of corresponding discrete passive currents (the dimension of the $I_{e}$ vector) is very large (in the order of 100 elements for the ITER tokamak).

Assuming that both, the active coil currents vector $I_{a}$ and the plasma current distribution parameters vector $w$ are known, equation (1) can be rewritten as

$$
\begin{aligned}
& L_{e, e} \dot{I}_{e}=-R_{e} I_{e}-L_{e, a} \dot{I}_{a}-L_{e, w} \dot{w} \\
& y=C_{e} I_{e}+C_{a} I_{a}+F w+d_{y}
\end{aligned}
$$

Where the vector $d_{y}$ denotes the additive measurement errors, modeling random noise, drifts and biases. Assuming as state variable

$$
x_{1}=I_{e}+L_{e, e}^{-1} L_{e, a} I_{a}+L_{e, e}^{-1} L_{e, w} w
$$

model (2) can be readily converted into a standard state-space model

$$
\begin{aligned}
& \dot{x}_{1}=A x_{1}+B u+B_{w} w_{1} \\
& \dot{x}_{2}=w_{2} \\
& y=C x_{1}+x_{2}+D u+F w_{1}
\end{aligned}
$$

where $u=I_{a}, w_{1}=w$, and $x_{2}$ are state variables representing additional integrators generating biases and drift on the outputs $y$ if fed by impulsive or constant disturbance input $w_{2}$.

A classical state observer dynamics is

$$
\begin{aligned}
& \dot{\hat{x}}_{1}=A \hat{x}_{1}+B u+B_{w} w_{1}+\hat{u} \\
& \dot{\hat{x}}_{2}=w_{2} \\
& \hat{y}=C \hat{x}_{1}+\hat{x}_{2}+D u+F w_{1} \\
& \hat{u}=L(y-\hat{y})
\end{aligned}
$$

where $L$ is the observer matrix gain. On the base of the $H_{\infty}$ filtering theory [8], the observer matrix gain $L$ can be evaluated in such a way to optimize a given performance index. Once the the estimated state has been obtained, the filtered measurements $\hat{y}-\hat{x}_{2}$, can be used to substitute vector $y$ as an input to the EFIT++ code. This filtered measurements vector will be partially washed out by the measurement noise (the level of the attenuation of the noise depends on the observer gain matrix) .

\section{Application to the ITER tokamak}

The ITER poloidal section with the magnetic sensors used for the plasma identification is depicted in Fig. 1. Sensors are divided in coils measuring magnetic fields (AA, AB, AL, A3-A6 sensors), and flux loops measuring fluxes (AD, AE, and A7 sensors). Some are internal to the vacuum vessel (70 inner vessel sensors in total) and others are external (166 outer vessel sensors in total). The internal sensors will be more affected by neutron induced drifts and faults being exposed to the nuclear environment during nuclear operational phases. On the other side the external sensors can guarantee a smaller bandwidth to the plasma due to the screen effect from the vacuum chamber $(0.5 \mathrm{~s})$.

Various sources of measurement errors have been modeled as Gaussian random signals. In particular we have constant multiplicative noise $n_{1}$, a time-varying multiplicative noise $n_{2}$, and two additive time varying noise signals $n_{3}$ and $n_{4}$, with $n_{4}$ representing the drift due to the offset at the input of the sensor integrators 


$$
y_{m}(t)=y(t)\left(1+n_{1}+n_{2}(t)\right)+n_{3}(t)+n_{4}(t)
$$

The standard deviation of each error term is reported in Table 1. For the drift term $n_{4}$ the standard deviation is referred to the slope $\left(\mathrm{mT} / \mathrm{s}\right.$ or $\left.\mathrm{mV} / \mathrm{s}^{2}\right)$.

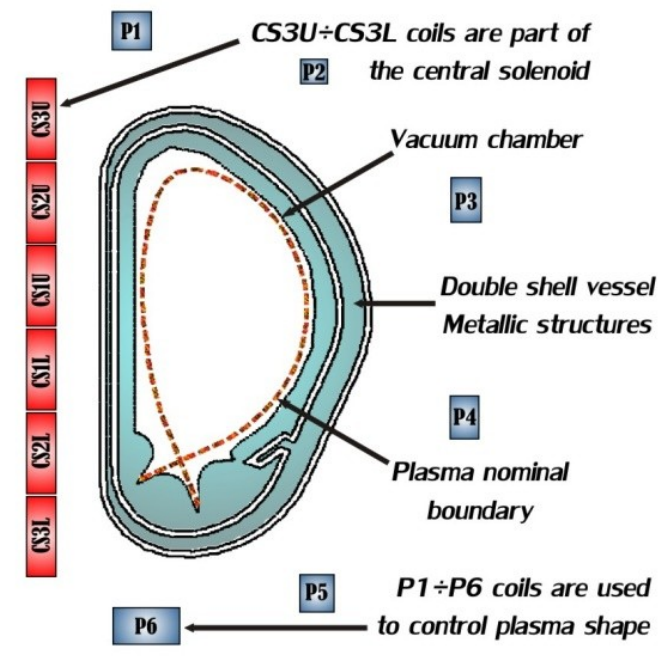

(a)

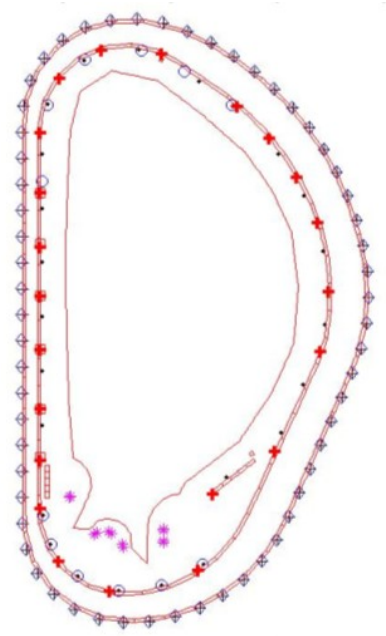

(b)

Fig. 1. (a) Schematic of the ITER tokamak. The eddy currents flow in the double shell vessel structure.

(b) Location of sensors in the poloidal section: AA (black dot), AB normal coils (blue o), AD (red +), AL (magenta *), A3 (blue diamonds) A4 (black +), A7 (red squares)

\begin{tabular}{|l|c|c|c|c|}
\hline Stdv & $n_{1}$ & $n_{2}$ & $n_{3}$ & $n_{4}$ \\
\hline$A A$ & $1.1 \mathrm{e}-3$ & $1 \mathrm{e}-3$ & $7.5 \mathrm{e}-4$ & $4.1 \mathrm{e}-3$ \\
\hline$A B$ & $1.1 \mathrm{e}-3$ & $1 \mathrm{e}-3$ & $7.5 \mathrm{e}-4$ & $3.9 \mathrm{e}-3$ \\
\hline$A D$ & 0 & $1 \mathrm{e}-3$ & 0 & $8.7 \mathrm{e}-4$ \\
\hline$A L$ & $1.1 \mathrm{e}-3$ & $1 \mathrm{e}-3$ & $7.5 \mathrm{e}-4$ & $1.0 \mathrm{e}-2$ \\
\hline$A 3$ & $1.0 \mathrm{e}-3$ & $1 \mathrm{e}-3$ & $7.5 \mathrm{e}-4$ & $5.0 \mathrm{e}-5$ \\
\hline$A 4$ & $1.0 \mathrm{e}-3$ & $1 \mathrm{e}-3$ & $7.5 \mathrm{e}-4$ & $5.0 \mathrm{e}-5$ \\
\hline$A 5$ & $1.0 \mathrm{e}-3$ & $1 \mathrm{e}-3$ & $7.5 \mathrm{e}-4$ & 0 \\
\hline$A 6$ & $1.0 \mathrm{e}-3$ & $1 \mathrm{e}-3$ & $7.5 \mathrm{e}-4$ & 0 \\
\hline$A 7$ & $8.5 \mathrm{e}-4$ & $1 \mathrm{e}-3$ & 0 & $4.9 \mathrm{e}-3$ \\
\hline$A E$ & $8.5 \mathrm{e}-4$ & $1 \mathrm{e}-3$ & 0 & $4.9 \mathrm{e}-3$ \\
\hline
\end{tabular}

Table 1. Noise main characteristics. The standard deviations in columns 2-4 are in $\mathrm{mT}$ for magnetic field sensors and $\mathrm{mVs}$ for flux sensors. The standard deviation in column 5 are in $\mathrm{mT} / \mathrm{s}$ for magnetic field sensors and $\mathrm{mV}$ for flux sensors.

After the design, the observer performance were validated on two different discharge segments extracted from the ITER Case\#001 Scenario studied in [6]. In particular we considered

a) the plasma current ramp-up phase;

b) the 15 MA flat top phase.

Segment a) was used to assess the capability of the observer to reconstruct eddy currents during the transient caused by the large plasma current ramp rate, whereas segment $b$ ) was used to assess the capability of the observer in reducing the noise level on the inner vessel sensors, by substituting the measured values coming from these sensors with their estimated values provided by the observer.

The data used for the simulation of the ITER scenario were obtained by using the CREATE-NL code [2]. A 110 states model, as described in equation (1), was used to simulate 
the scenario segments. In order to reduce the computation burden and to demonstrate the robustness of the proposed approach to modeling errors and uncertainties, the circuits dynamic model was reduced from 110 to 30 states using the balanced truncation model reduction available in Matlab. Moreover random uncertainties in the order of $0.1 \%$ of the nominal values were added to all elements of the matrices of equation (1). Finally an uncertainty of $0.1 \%$ on the plasma current profile was considered.

Fig. 2 shows the plasma current, the main plasma parameters, the eddy currents, and the eddy current estimation error during the ramp-up phase simulation.

Fig. 3 shows the estimation error on six plasma wall gaps, when using as EFIT++ inputs the measurement from the outer vessel sensors and the estimated measurement of the inner vessel sensors during the flat top phase. As it is shown in Fig. 3, the plasma wall-gap estimation errors are well below $1 \mathrm{~cm}$, the same order of magnitude obtained with the simulation in the absence of eddy currents. The error estimating the gaps neglecting the eddy currents and without the correction provided by the observer can instead be larger than $1 \mathrm{~cm}$.

The simulation of the flat-top phase is also demonstrating how the model can account for hard faults on the sensors system, as is in the case of the failure of the entire in-vessel sensor set. The performance of the observer guarantees an accuracy for the plasma parameters estimation within the requirements.

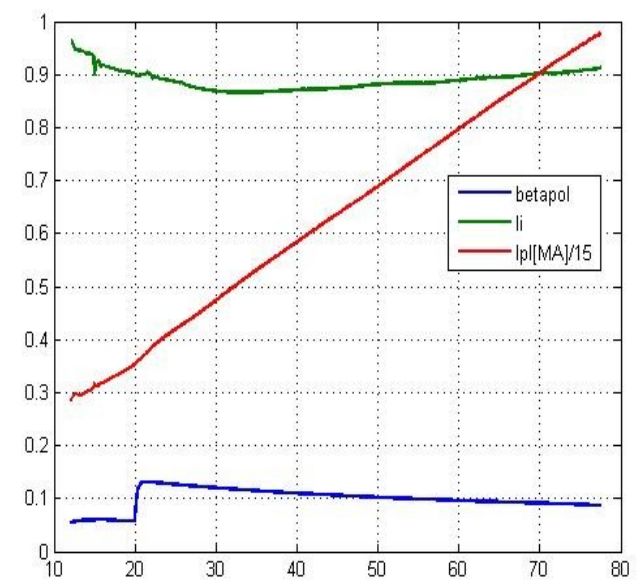

(a)

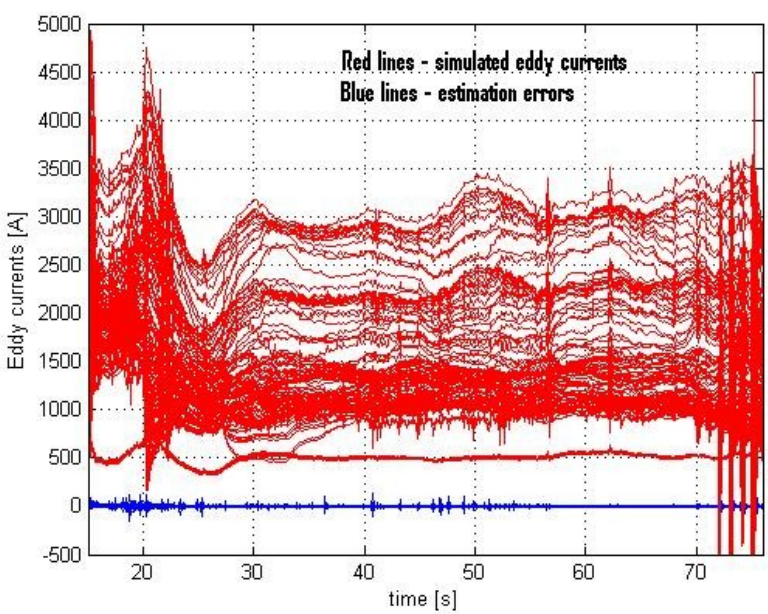

(b)

Fig. 2. (a) Time evolution of the of the main plasma parameters . (b) Time evolution of the 110 eddy currents (red lines), and of the eddy currents estimation errors (blue lines)

\section{Conclusions}

An $\mathrm{H}_{\infty}$ observer to estimate eddy currents in the passive structures of a tokamak have been tested in view of a possible application to ITER. The estimation has been oriented to the plasma shape identification using magnetic probes in the presence of random noise, biases, and drifts on the magnetic measurements. The proposed filter allows an accurate estimation also in the presence of faults of part of the magnetic sensors. In particular the failure of all the sensors internal to the tokamak vessel has been successfully managed to achieve satisfactory plasma shape identifications. The performance of the observer has also been tested on a plasma current ramp up where the eddy currents distribution in the passive structure is well reconstructed also during fast transients. Future work will be devoted to increase the observer robustness in the 
presence of structured uncertainties on inductance, resistance and input-output system matrices. An extensive campaign of simulation on different kind of scenarios will also be made.

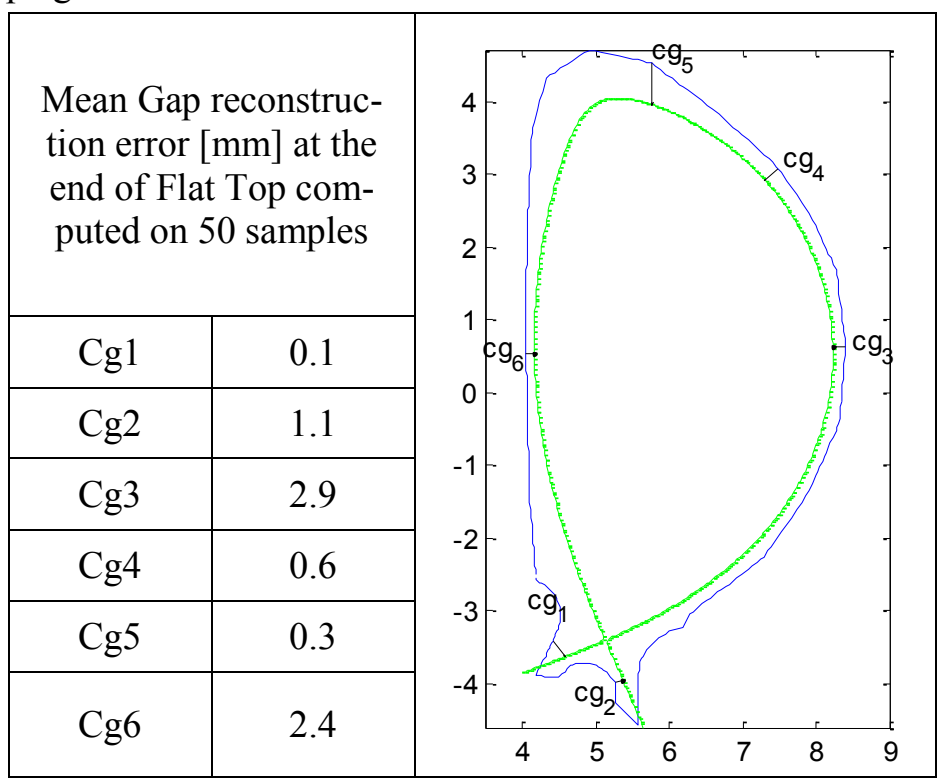

Fig. 3. Effect of the estimation errors on the plasma shape reconstruction using EFIT++

\section{References}

[1] Control Systems Magazine, Special Issue on the Control of tokamak plasmas, Vol.25, Issue 5, 2005.

[2] R. Albanese, R., G. Artaserse, et al., Plasma Reconstruction in Tokamaks with Linearized Approaches, International Journal of Applied Electomagnetics and Mechanics, Vol. 26, 191-199, 2007.

[3] R. Albanese, G. Ambrosino, Magnetic control of plasma current, position, and shape in Tokamaks: a survey or modeling and control approaches, Control Systems Magazine, Vol.25, Issue 5, 76-92, 2005.

[4] R. Albanese, R. Ambrosino,et.al, Open loop optimal control for plasma scenarios in the presence of eddy currents, Proc. of the 2011 IEEE Multi-Conference on Systems \& Control, Denver, September 2011

[5] L. C. Appel, G.T.A. Huysman, et al., A Unified Approach to, Equilibrium Reconstruction, Proc. Of 33rd EPS Conference on Plasma Physics, Rome, Italy, 2006.

[6] F. Koechl, V. Parail, et al., Self-consistent predictive modelling of 15 MA inductive scenarios in ITER, 38th EPS conference on Plasma Physics, Strasbourg, June 2011.

[7] Lao, L. H. St. John, et al., Reconstruction of current profile parameters and plasma shapes in tokamaks, Nuclear Fusion, Vol. 25, n. 8, 1611-1615, 1985.

[8] K. M. Nagpal, K. K. Khargonekar, Filtering and smoothing in an $H_{\infty}$ setting, IEEE Transactions on Automatic Control, vol. 36, n. 2, 1991. 